\title{
Subinhibitory concentrations of moxifloxacin decrease adhesion and biofilm formation of Stenotrophomonas maltophilia from cystic fibrosis
}

Correspondence

G. Di Bonaventura

gdibonaventura@unich.it

Received 23 April 2009

Accepted 16 September 2009
A. Pompilio, ${ }^{1,2}$ C. Catavitello, ${ }^{3}$ C. Picciani, ${ }^{1,2}$ P. Confalone, ${ }^{1,2}$ R. Piccolomini, ${ }^{1,2}$ V. Savini, ${ }^{3}$ E. Fiscarelli, ${ }^{4}$ D. D'Antonio ${ }^{3}$ and G. Di Bonaventura ${ }^{1,2}$

\author{
${ }^{1}$ Clinical Microbiology Unit, Department of Biomedical Sciences, 'G. D'Annunzio' University of \\ Chieti-Pescara, Chieti, Italy \\ ${ }^{2}$ Center for Excellence on Aging, 'G. D'Annunzio' University Foundation, Chieti, Italy \\ ${ }^{3}$ Clinical Microbiology and Virology Unit, Department of Transfusion Medicine, 'Spirito Santo' \\ Hospital, Pescara, Italy \\ ${ }^{4}$ Laboratory of Clinical Microbiology, 'Bambino Gesù' Pediatric Hospital, Rome, Italy
}

\section{INTRODUCTION}

Stenotrophomonas maltophilia is an emerging nosocomial pathogen, especially in the immunocompromised host. Although recent clinical evidence suggests an increase in the frequency of isolation of the micro-organism from the respiratory tract of patients with cystic fibrosis (CF) (Conway et al., 2003), the role of S. maltophilia in the pathophysiology of CF lung disease has not yet been clearly elucidated (O'Sullivan \& Freedman, 2009; Ryan et al., 2009). However, the reported isolation of S. maltophilia from the sputa of CF patients has become a cause for concern in the CF community, as the organism is resistant to many of the antibiotics prescribed in the management of CF (Nicodemo \& Paez, 2007).

The effective therapeutic result of antibiotics is best when the concentration is above the MIC. However, after a certain period of time following a dose, antibiotic concentrations within many tissues become lower than

Abbreviations: ANOVA, analysis of variance; CF, cystic fibrosis; $\mathrm{MATH}$, microbial adherence to $\mathrm{n}$-hexadecane; subMIC, subinhibitory concentration. the MIC and are called subinhibitory concentrations (subMICs). Micro-organisms often grow in the presence of subMICs, which, although not able to inactivate microorganisms, are potentially capable of altering the chemical and physical cell-surface characteristics and consequently the functionality and expression of some virulence properties such as adhesion, biofilm formation, hydrophobicity and motility (Fonseca et al., 2004; Wojnicz \& Jankowski, 2007).

Most studies on the effects of subMICs of antibiotics have focused largely on Escherichia coli (Woinicz \& Jankowski, 2007), Staphylococcus spp. (Chisari et al., 2002; Cerca et al., 2005), and Pseudomonas aeruginosa (Fonseca et al., 2004), and, to the best of our knowledge, there is no such information in the literature concerning S. maltophilia.

Infections caused by S. maltophilia are particularly difficult to eradicate because it is usually highly resistant to many of the currently available broad-spectrum antibiotics (Avison et al., 2001; Gould \& Avison, 2006). Moxifloxacin, a recent fluoroquinolone used mainly in the treatment of respiratory infections, exhibits significant in vitro activity against S. maltophilia (Galles et al., 2008), including strains 
resistant to trimethoprim/sulfamethoxazole (GiamarellosBourboulis et al., 2002), the drug of choice for the treatment of infections, thus indicating that this quinolone should be evaluated further as a therapeutic option for $S$. maltophilia infections. Considering the potential clinical implications, we performed the present study in an attempt to evaluate the effect of moxifloxacin subMICs against a number of virulence properties (adhesion, biofilm formation and cell-surface hydrophobicity) expressed by $S$. maltophilia isolated from CF patients.

\section{METHODS}

Bacterial strains and culture conditions. The S. maltophilia Sm132 and Sm144 strains used in this study were collected from the respiratory tract of two different patients with $\mathrm{CF}$ admitted to the Bambino Gesù Pediatric Hospital, and who had not been treated previously with moxifloxacin. Identification of S. maltophilia was performed by biochemical tests (BBL Crystal E/NF; Becton Dickinson) and confirmed by rRNA-directed PCR (Whitby et al., 2000). Both strains were selected for their ability to form significant amounts of biofilm on polystyrene. Stock cultures were maintained at $-80{ }^{\circ} \mathrm{C}$ in a Microbank preservation system (Pro-Lab Diagnostics) until use. Stocks were thawed as needed and subcultured twice on Mueller-Hinton agar (MHA; Oxoid) for $24 \mathrm{~h}$ at $37^{\circ} \mathrm{C}$.

Antimicrobial susceptibility. Reagent-grade moxifloxacin powder of known potency was kindly supplied by Bayer (Milan, Italy). Moxifloxacin MICs for the S. maltophilia Sm132 and Sm144 strains were determined using a broth microdilution technique according to Clinical and Laboratory Standards Institute guidelines (CLSI, 2006). Results were observed after overnight incubation at $37{ }^{\circ} \mathrm{C}$, with the MIC defined as the lowest concentration that inhibited visible growth. $P$. aeruginosa ATCC 27853 was included in each run of experiments as a quality control.

Bacterial exposure to moxifloxacin at subMICs. Briefly, colonies from an overnight culture grown on MHA were suspended in trypticase soy broth (TSB; Oxoid) at a concentration of approximately $1 \times 10^{8}$ c.f.u. $\mathrm{ml}^{-1}$, diluted $1: 10$ in cation-adjusted MuellerHinton broth (Becton Dickinson) and then exposed $\left(37^{\circ} \mathrm{C}, 18 \mathrm{~h}\right)$, under static conditions, to moxifloxacin at concentrations of $0.06 \times$ and $0.03 \times$ MIC. Control samples consisted of bacteria that were not exposed to moxifloxacin but were otherwise treated identically. Bacteria were then washed three times in PBS $\left(10500 \mathrm{~g}, 5 \mathrm{~min}, 4{ }^{\circ} \mathrm{C}\right)$ to remove the antibiotic and resuspended in PBS (for the hydrophobicity assay) or TSB (for adhesion and biofilm formation assays) to an $\mathrm{OD}_{550}$ of 0.80 or 0.13 , respectively. The inoculum concentration was determined by making serial dilutions in PBS and performing colony counts in duplicate on MHA after overnight incubation at $37^{\circ} \mathrm{C}$.

Adhesion to and biofilm formation on polystyrene. One millilitre of the standardized inoculum was dispensed into each well of a sterile, flat-bottomed, polystyrene, 24-well, tissue culture-treated culture plate (Iwaki Glass). After $3 \mathrm{~h}$ (adhesion) or $24 \mathrm{~h}$ (biofilm formation) of static incubation at $37{ }^{\circ} \mathrm{C}$, the medium containing non-adherent cells was removed and each well was washed carefully three times with $1 \mathrm{ml}$ sterile PBS ( $\mathrm{pH}$ 7.3; Sigma-Aldrich), previously warmed at room temperature, using a micropipette. To avoid inter-operator variability, sample washings were carried out by the same operator. Adherent and biofilm cells were then harvested by scraping and quantified by colony counts.
Cell-surface hydrophobicity. Evaluation of hydrophobicity was carried out by using a microbial adherence to n-hexadecane (MATH) test (Mattos-Guaraldi et al., 1999). Briefly, $4 \mathrm{ml}$ moxifloxacinexposed inoculum prepared in $\mathrm{PBS}$ at an $\mathrm{OD}_{550}$ of 0.8 was overlaid with $400 \mu \mathrm{n}$-hexadecane (Sigma-Aldrich). After $1 \mathrm{~min}$ agitation by vortexing, the phases were allowed to separate for $15 \mathrm{~min}$ at room temperature. The $\mathrm{OD}_{500}$ value of the aqueous phase was then measured. The results were expressed as the proportion of cells that were excluded from the aqueous phase, determined by the equation: $\left[\left(A_{0}-A\right) / A_{0}\right] \times 100$, where $A_{0}$ and $A$ are, respectively, the initial and final $\mathrm{OD}_{500}$ of the aqueous phase. Strains were considered as strongly hydrophobic when the values obtained were $>50 \%$, moderately hydrophobic for values ranging between 20 and $50 \%$, and hydrophilic when values were $<20 \%$ (Mattos-Guaraldi et al., 1999).

Bacterial morphology. The effects of moxifloxacin subMICs on $S$. maltophilia cell morphology were assessed by scanning electron microscopy. Briefly, samples were fixed for $3 \mathrm{~h}$ at $4{ }^{\circ} \mathrm{C}$ with $2.5 \%(\mathrm{v} /$ v) glutaraldehyde in $0.15 \mathrm{M}$ sodium cacodylate buffer ( $\mathrm{pH} 7.4)$, with the cationic dye ruthenium red $(0.15 \%$, w/v; Polysciences Europe). Samples were then post-fixed in $1.5 \%(\mathrm{v} / \mathrm{v}) \mathrm{OsO}_{4}$, rinsed in $0.15 \mathrm{M}$ cacodylate buffer and dehydrated in an ascending ethanol series (30, 50, 70, 80, 95 and $100 \%, \mathrm{v} / \mathrm{v})$ before critical-point drying. Examination of samples was performed at $15 \mathrm{kV}$ in a Hitachi S-500 scanning electron microscope.

Data analysis. Each experiment was carried out in triplicate and repeated on at least two different occasions; the results are presented as means \pm SD. Data from the adhesion and biofilm assays were normalized to the control, which was taken as $100 \%$. All data were statistically analysed by one-way analysis of variance (ANOVA), followed by pairwise comparisons using the Bonferroni test. The difference was considered statistically significant for $P<0.05$. Analyses were performed using the Prism 5.0 software package (GraphPad Software).

\section{RESULTS AND DISCUSSION}

\section{Susceptibility to moxifloxacin and determination of subMICs}

Both strains of S. maltophilia tested were susceptible to moxifloxacin (MIC $0.5 \mu \mathrm{g} \mathrm{ml} \mathrm{m}^{-1}$ for both strains). Preliminary studies were conducted in order to assess the killing of $S$. maltophilia following exposure to moxifloxacin at different subMICs $(0.5 \times, 0.25 \times, 0.12 \times, 0.06 \times$ and $0.03 \times$ MIC). Exposure to moxifloxacin at $0.5 \times, 0.25 \times$ and $0.12 \times$ MIC caused significant killing (ranging from 43.0 to $94.0 \%$ for the Sm132 strain and from 45.8 to $81.7 \%$ for the Sm144 strain), compared with the control (without moxifloxacin). No killing was observed after exposure to $0.06 \times$ and $0.03 \times$ MIC. Thus, all experiments carried out in this study were performed using a standardized S. maltophilia inoculum exposed to moxifloxacin at $0.06 \times$ and $0.03 \times \mathrm{MIC}$, corresponding to 0.03 and $0.015 \mu \mathrm{g} \mathrm{ml}^{-1}$, respectively.

\section{Effect of moxifloxacin on bacterial adherence}

The mean adherence of $S$. maltophilia to polystyrene after exposure to subMICs of moxifloxacin is shown in Fig. 1. Adhesion by Sm132 strain was, in comparison with the 


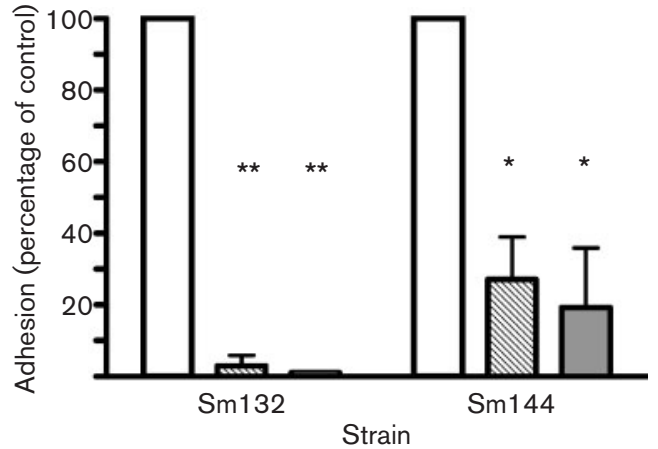

Fig. 1. Effect of moxifloxacin subMICs on adherence of $S$. maltophilia strains Sm132 and Sm144 to polystyrene. The control contained bacteria that were not exposed to moxifloxacin. Results are expressed as means $\pm \mathrm{SD} .{ }^{*}, P<0.05 ;{ }^{*}, P<0.001$ (versus control) (ANOVA+Bonferroni's post-test). White bars, control; hatched bars, $0.03 \times \mathrm{MIC}$; grey bars, $0.06 \times \mathrm{MIC}$.

control, significantly lower $(P<0.001)$ in the presence of $0.03 \times$ and $0.06 \times$ MIC, with inhibition levels of $97.0 \pm 2.9$ and $98.7 \pm 0.2 \%$, respectively. Adhesion by Sm144 strain was, in comparison with the control, also significantly lower $(P<0.05)$ in the presence of $0.03 \times$ and $0.06 \times$ MIC, with inhibition levels of $72.9 \pm 11.8$ and $80.7 \pm 16.6 \%$, respectively. No statistically significant differences were observed between inhibition levels caused by $0.06 \times$ and $0.03 \times$ MIC exposure for either strain.

The ability to adhere to abiotic and biotic substrates is a prerequisite for infection so that the micro-organism can colonize and thus cause infection in the host. One of the major risk factors associated with S. maltophilia is the presence of prosthetic devices (Muder et al., 1996). We recently demonstrated that the ability to adhere to polystyrene is highly conserved in S. maltophilia isolates from CF patients (Di Bonaventura et al., 2007).

SubMICs of antibiotics could inhibit bacterial adhesion through different mechanisms. They may inhibit the synthesis or expression of adhesins on the bacterial cell surface, lead to the formation of functionally aberrant adhesins, cause the release of adhesins from the surface of bacterial cells or modify the bacterial shape in a such way as to interfere with the ability of the micro-organisms to approach host cell-surface receptors (Lorian \& Ernst, 1987; Lorian et al., 1989).

These data confirm the anti-adhesive properties of fluoroquinolones in general (Baskin et al., 2002; Wojnicz \& Jankowski, 2007). Indeed, moxifloxacin caused a significant reduction in $S$. maltophilia adherence up to concentrations equal to $0.03 \times$ MIC, unlike previous studies with $E$. coli in which the effect of fluoroquinolones was limited at concentrations equal to or not less than $0.125 \times$ MIC (Baskin et al., 2002; Wojnicz \& Jankowski, 2007).

\section{Effect of moxifloxacin on biofilm formation}

The effects of subMICs of moxifloxacin on S. maltophilia biofilm formation on polystyrene are shown in Fig. 2. Biofilm production by $\mathrm{Sm} 132$ strain was, in comparison with the control, significantly lower $(P<0.05)$ in the presence of $0.03 \times$ and $0.06 \times$ MIC, with inhibition levels of $65.4 \pm 11.2$, and $65.3 \pm 37.1 \%$, respectively. Biofilm production by $S m 144$ strain was, in comparison with the control, also significantly lower $(P<0.001)$ in the presence of $0.03 \times$ and $0.06 \times \mathrm{MIC}$, with inhibition levels of $74.8 \pm 14.2$, and $72.3 \pm 7.3 \%$, respectively. No statistically significant differences were observed between inhibition levels caused by $0.06 \times$ and $0.03 \times$ MIC exposure for either strain.

A number of studies have demonstrated that, in CF patients, the inefficiency of antibiotics used to treat infections is related to the ability of the micro-organisms to form biofilms (Costerton, 2001; Ebert \& Olivier, 2002). The finding that adhesion and biofilm formation are highly conserved in S. maltophilia isolates from CF patients (Di Bonaventura et al., 2007, 2008b) has led to the suggestion that in vivo this micro-organism may also develop biofilms, which may protect it from natural or acquired immune defences and from the action of antibiotics. Recently, we observed that $S$. maltophilia is able to form biofilms on CF bronchial epithelial cells (Di Bonaventura et al., 2008a), thus providing a rationale for the persistence of the bacterium in CF patients. The results in Fig. 2 demonstrate that exposure to moxifloxacin subMICs is associated with a significant decrease in the ability of S. maltophilia to form biofilms on polystyrene, which may leave cells more susceptible to immune clearance or antimicrobial drug therapy.

Although having the same mechanism of action as that of moxifloxacin, other quinolones show anti-biofilm prop-

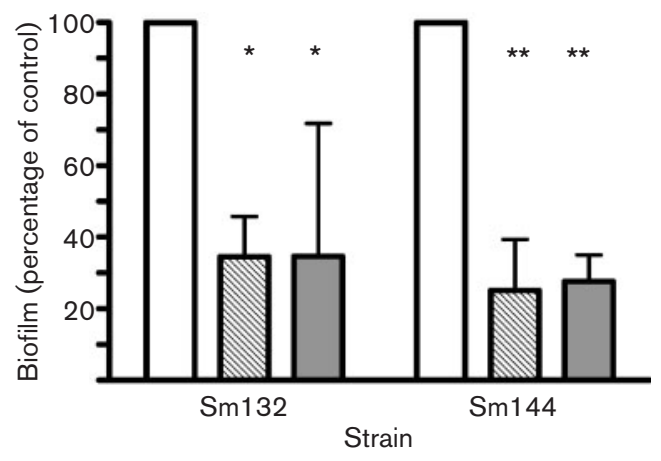

Fig. 2. Effect of moxifloxacin subMICs on biofilm formation of $S$. maltophilia strains Sm132 and Sm144 on polystyrene. The control contained bacteria that were not exposed to moxifloxacin. Results are expressed as means $\pm \mathrm{SD} .{ }^{*}, P<0.05 ;{ }^{* *}, P<0.001$ (versus control) (ANOVA + Bonferroni's post-test). White bars, control; hatched bars, $0.03 \times \mathrm{MIC}$; grey bars, $0.06 \times \mathrm{MIC}$. 
erties to a lesser extent at subMICs. In fact, we found previously that moxifloxacin was the most active fluoroquinolone in affecting $S$. maltophilia biofilm formation at $0.5 \times$ and $0.25 \times \mathrm{MIC}$, being about twice as active as levofloxacin and grepafloxacin, whilst norfloxacin was less active among the fluoroquinolones tested (Di Bonaventura et al., 2004). Thus, we hypothesize that the significant antibiofilm activity of moxifloxacin subMICs is a specific property not shared by other antibiotics with similar structures and mechanisms of action.

\section{Effect of moxifloxacin on cell-surface hydrophobicity}

The effect of moxifloxacin subMICs on cell-surface hydrophobicity is summarized in Fig. 3. In particular, the presence of moxifloxacin did not significantly affect the MATH of strain Sm132. which preserved its original hydrophilicity (MATH $6.6 \pm 5.0,2.9 \pm 2.9$ and $4.8 \pm 3.4 \%$ for the control, $0.03 \times$ and $0.06 \times$ MIC, respectively; $P>0.05)$. Strain Sm144, which started off with a hydrophobic MATH in the absence of moxifloxacin $(25.9 \pm 3.2 \%)$, preserved its hydrophobicity in the presence of moxifloxacin at $0.03 \times \mathrm{MIC}(21.1 \pm 4.8 \% ; P>0.05$ vs control), but became hydrophilic in the presence of moxifloxacin at $0.06 \times \mathrm{MIC}$, as indicated by the statistically significant decrease in MATH with respect to the control (16.9 $\pm 3.7 \% ; P<0.001$ vs control).

As soon as micro-organisms reach a living (epithelial mucosa) or non-living (prostheses) surface, they will be attracted or repelled by it, depending on the sum of the different non-specific interactions, especially by hydrophobic interactions (Doyle, 2000; Costa et al., 2006). Recently, we observed a positive correlation between hydrophobicity and levels of both adhesion and biofilm

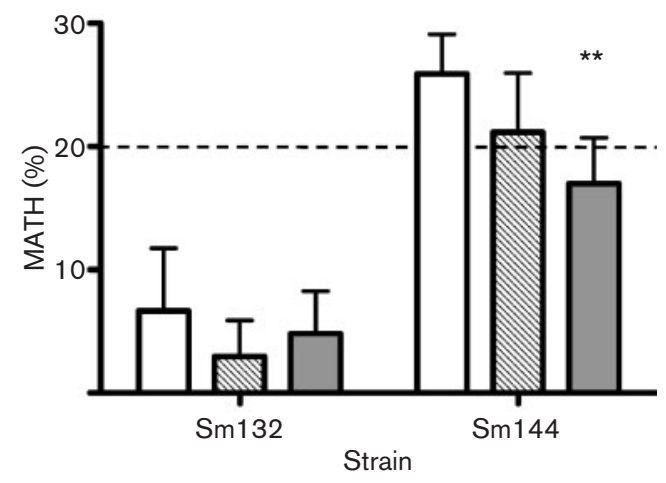

Fig. 3. Effects of moxifloxacin subMICs on hydrophobicity of $S$. maltophilia strains Sm132 and Sm144. The dotted line indicates the cut-off point for hydrophobicity (MATH $>20 \%$ ). The control contained bacteria that were not exposed to moxifloxacin. Results are expressed as means $\pm \mathrm{SD} .{ }^{* *}, P<0.001$ (versus control) (ANOVA \pm Bonferroni's post-test). White bars, control; hatched bars, $0.03 \times \mathrm{MIC}$; grey bars, $0.06 \times \mathrm{MIC}$. formation in S. maltophilia isolates from neutropenic patients (Pompilio et al., 2008), thus suggesting that hydrophobicity is a significant determinant of adhesion and biofilm formation by S. maltophilia.

The results of the present study suggest that moxifloxacin can modulate the level of hydrophobicity in S. maltophilia, although this effect appears to be strain-dependent.

Moxifloxacin could decrease cell-surface hydrophobicity by interfering with the synthesis and expression of outermembrane proteins, LPS or fimbriae, structures known to affect bacterial hydrophobicity (Doyle, 2000; Costa et al., 2006; Pompilio et al., 2008). However, further studies are needed to clarify whether the reduction in hydrophobicity levels induced by moxifloxacin can be extended to a larger number of strains.

\section{Effect of moxifloxacin on bacterial morphology}

SubMICs of antibiotics, particularly fluoroquinolones, can reduce the virulence of some bacteria (Gram-negative bacilli, in particular) by inducing an elongation of the cell soma along its longitudinal axis, a phenomenon also known as filamentation. These morphologically altered cells generally show reduced pathogenicity in terms of lower levels of adhesion, altered susceptibility to phagocytosis and decreased release of bacterial enzymes (Labro et al., 1987; Chen et al., 2005). Recently, Drago et al. (2005) observed that moxifloxacin subMICs $(0.125 \times$ and $0.06 \times$ MIC) induced filamentation in a remarkable portion of Klebsiella pneumoniae in an animal experimental model of pulmonary infection.

On this basis, we examined the effect of exposure to moxifloxacin at $0.06 \times$ and $0.03 \times$ MIC on S. maltophilia morphology. Electron microscopic analysis revealed that moxifloxacin subMICs did not induce morphological abnormalities, as showed by the absence of such extrusions in the cell envelope in the form of blebs or bacterial filamentation (Fig. 4).

In conclusion, our results showed that moxifloxacin subMICs were effective in preventing initial adherence and subsequent biofilm formation by $S$. maltophilia, possibly by at least two mechanisms: a cell-surface hydrophobicity-dependent and a cell-surface hydrophobicity-independent mechanism.

After a daily oral dose of $400 \mathrm{mg}$, moxifloxacin reaches a peak serum level of $3.3 \mu \mathrm{g} \mathrm{ml}^{-1}$, attaining $5.5 \mu \mathrm{g} \mathrm{ml}^{-1}$ in bronchial mucosa and $24.4 \mu \mathrm{g} \mathrm{ml}^{-1}$ in the epithelial lining fluid (Schubert et al., 2005). As we found that the antiadhesive effect produced by moxifloxacin persists up to $0.015 \mu \mathrm{g} \mathrm{ml}^{-1}$ (corresponding to $0.03 \times$ MIC for both strains tested), it is plausible that clinically attainable moxifloxacin concentrations would inhibit S. maltophilia adherence and biofilm formation, even if the cells are resistant to killing at these concentrations.

Thus, results of the present study could have important clinical implications, providing an additional rationale for 

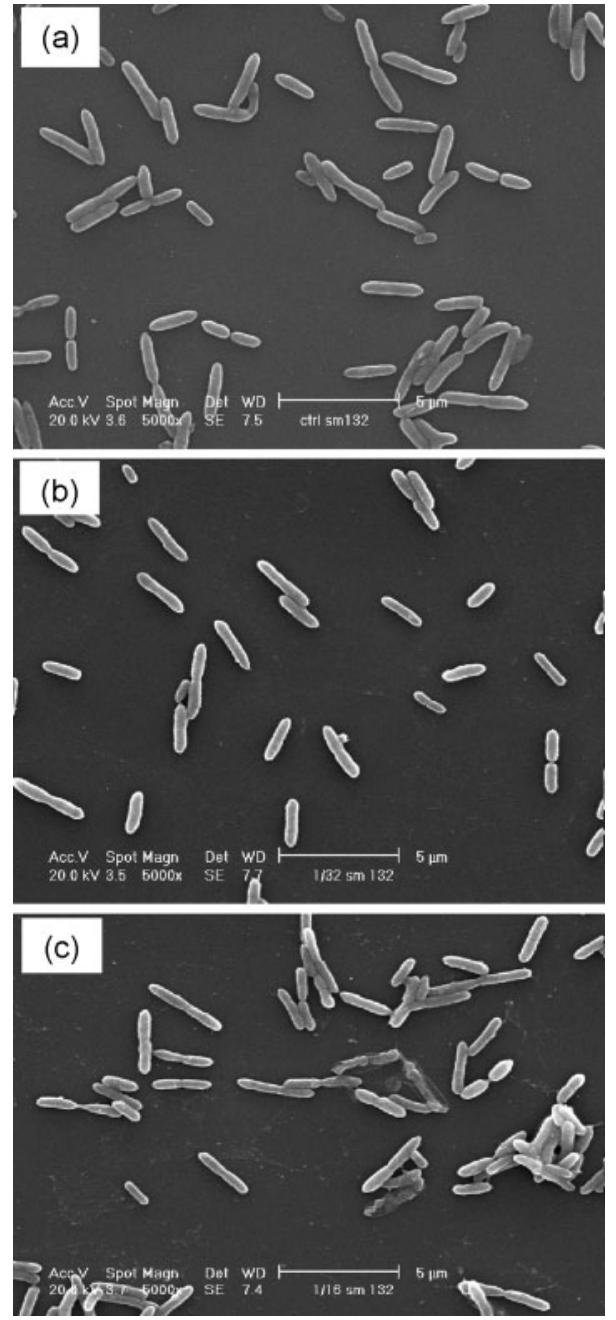

Fig. 4. Scanning electron microscopy of S. maltophilia Sm132 strain in the absence (a) and presence of $0.03 \times \mathrm{MIC}(\mathrm{b})$ or $0.06 \times$ MIC (c) moxifloxacin for $18 \mathrm{~h}$. Bars, $5 \mu \mathrm{m}$. Micrographs are also representative of the results obtained for S. maltophilia Sm144.

the use of moxifloxacin in CF patients and more generally in biofilm-related infections involving $S$. maltophilia. Future work is needed to confirm our findings in an animal model.

\section{ACKNOWLEDGEMENTS}

This study was supported by the Italian Cystic Fibrosis Research Foundation (grant \#7/2007).

\section{REFERENCES}

Avison, M. B., Higgings, C. S., von Heldreich, C. J., Bennett, P. M. \& Walsh, T. R. (2001). Plasmid location and molecular heterogeneity of the L1 and L2 $\beta$-lactamase genes of Stenotrophomonas maltophilia. Antimicrob Agents Chemother 45, 413-419.
Baskin, H., Doğan, Y., Bahar, I. H. \& Yuluğ, N. (2002). Effect of subminimal inhibitory concentrations of three fluoroquinolones on adherence of uropathogenic strains of Escherichia coli. Int $J$ Antimicrob Agents 19, 79-82.

Cerca, N., Martins, S., Sillankorva, S., Jefferson, K. K., Pier, G. B., Oliveira, R. \& Azeredo, J. (2005). Effects of growth in the presence of subinhibitory concentrations of dicloxacillin on Staphylococcus epidermidis and Staphylococcus haemolyticus biofilms. Appl Environ Microbiol 71, 8677-8682.

Chen, K., Sun, G. W., Chua, K. L. \& Gan, Y. H. (2005). Modified virulence of antibiotic-induced Burkholderia pseudomallei filaments. Antimicrob Agents Chemother 49, 1002-1009.

Chisari, G., Accossano, S. \& Reibaldi, M. (2002). Effect of aminoglycosides (sub-MICs) on adherence of coagulase-negative staphylococci to intraocular lens surface. J Chemother 14, 574-578.

CLSI (2006). Methods for Dilution Antimicrobial Susceptibility Tests for Bacteria that Grow Aerobically, 7th edn, approved guideline M7-A7. Wayne, PA: Clinical and Laboratory Standards Institute.

Conway, S. P., Brownlee, K. G., Denton, M. \& Peckham, D. G. (2003). Antibiotic treatment of multidrug-resistant organisms in cystic fibrosis. Am J Respir Med 2, 321-332.

Costa, G. F., Tognim, M. C., Cardoso, C. L., Carrara-Marrone, F. E. \& Garcia, L. B. (2006). Preliminary evaluation of adherence on abiotic and cellular surfaces of Acinetobacter baumannii strains isolated from catheter tips. Braz J Infect Dis 10, 346-351.

Costerton, J. W. (2001). Cystic fibrosis pathogenesis and the role of biofilms in persistent infection. Trends Microbiol 9, 50-52.

Di Bonaventura, G., Spedicato, I., D’Antonio, D., Robuffo, I. \& Piccolomini, R. (2004). Biofilm formation by Stenotrophomonas maltophilia: modulation by quinolones, trimethoprim-sulfamethoxazole, and ceftazidime. Antimicrob Agents Chemother 48, 151-160.

Di Bonaventura, G., Prosseda, G., Del Chierico, F., Cannavacciuolo, S., Cipriani, P., Petrucca, A., Superti, F., Ammendolia, M. G., Concato, C. \& other authors (2007). Molecular characterization of virulence determinants of Stenotrophomonas maltophilia strains isolated from patients affected by cystic fibrosis. Int J Immunopathol Pharmacol 20, 529-537.

Di Bonaventura, G., Pompilio, A., Picciani, C., Nicoletti, M., Zappacosta, R. \& Piccolomini, R. (2008a). Adhesion to and biofilm formation on IB3-1 bronchial cells by Stenotrophomonas maltophilia: implications in cystic fibrosis. In 18th European Congress of Clinical Microbiology and Infectious Diseases, Barcelona, Spain, April 2008, poster no. 704.

Di Bonaventura, G., Pompilio, A., Picciani, C., Pomponio, S., Verginelli, F., Di Risio, A., Mongiana, M., Gherardi, G., Fiscarelli, E. $\&$ other authors (2008b). Phenotypic and genotypic characterization of biofilm formation by Stenotrophomonas maltophilia isolated from patients with cystic fibrosis. In Proceedings of 36th Congress of the Italian Society of Microbiology, Rome, Italy, October 2008. Poster no. 14.

Doyle, R. J. (2000). Contribution of the hydrophobic effect to microbial infection. Microbes Infect 2, 391-400.

Drago, L., De Vecchi, E., Nicola, L. \& Gismondo, M. R. (2005). Evaluation of antibacterial in vitro activity of moxifloxacin and its effects on pulmonary clearance of Klebsiella pneumoniae in an animal experimental model. Arzneimittelforschung 55, 473-477.

Ebert, D. L. \& Olivier, K. N. (2002). Nontuberculous mycobacteria in cystic fibrosis. Infect Dis Clin North Am 16, 221-233.

Fonseca, A. P., Extremina, C., Fonseca, A. F. \& Sousa, J. C. (2004). Effect of subinhibitory concentration of piperacillin/tazobactam on Pseudomonas aeruginosa. J Med Microbiol 53, 903-910.

Galles, A. C., Jones, R. N. \& Sader, H. S. (2008). Antimicrobial susceptibility profile of contemporary clinical strains of 
Stenotrophomonas maltophilia isolates: can moxifloxacin activity be predicted by levofloxacin MIC results? J Chemother 20, $38-42$.

Giamarellos-Bourboulis, E. J., Karnesis, L., Galani, I. \& Giamarellou, H. (2002). In vitro killing effect of moxifloxacin on clinical isolates of Stenotrophomonas maltophilia resistant to trimethoprim-sulfamethoxazole. Antimicrob Agents Chemother 46, 3997-3999.

Gould, V. C. \& Avison, M. B. (2006). SmeDEF-mediated antimicrobial drug resistance in Stenotrophomonas maltophilia clinical isolates having defined phylogenetic relationships. J Antimicrob Chemother 57, 1070-1076.

Labro, M. T., Pochet, I., Babin-Chevaye, C. \& Hakim, J. (1987). Effect of ceftriaxone-induced alterations of bacteria on neutrophil bactericidal function. J Antimicrob Chemother 20, 857-869.

Lorian, V. \& Ernst, J. (1987). Effects of antibiotic on bacterial structure and their pathogenicity. Pathol Biol 35, 1370-1376.

Lorian, V., Ernst, J. \& Amaral, L. (1989). The post-antibiotic effect defined by bacterial morphology. J Antimicrob Chemother 23, 485491.

Mattos-Guaraldi, A. L., Formiga, L. C. \& Andrade, A. F. (1999). Cell surface hydrophobicity of sucrose fermenting and nonfermenting Corynebacterium diphtheriae strains evaluated by different methods. Curr Microbiol 38, 37-42.

Muder, R. R., Harris, A. P., Muller, S., Edmond, M., Chow, J. W., Papadakis, K., Wagener, M. W., Bodey, G. P. \& Steckelberg, J. M. (1996). Bacteremia due to Stenotrophomonas (Xanthomonas) maltophilia: a prospective, multicenter study of 91 episodes. Clin Infect Dis 22, 508-512.

Nicodemo, A. C. \& Paez, J. I. (2007). Antimicrobial therapy for Stenotrophomonas maltophilia infections. Eur J Clin Microbiol Infect Dis 26, 229-237.

O'Sullivan, B. P. \& Freedman, S. D. (2009). Cystic fibrosis. Lancet 373, 1891-1904.

Pompilio, A., Piccolomini, R., Picciani, C., D’Antonio, D., Savini, V. \& Di Bonaventura, G. (2008). Factors associated with adherence to and biofilm formation on polystyrene by Stenotrophomonas maltophilia: the role of cell surface hydrophobicity and motility. FEMS Microbiol Lett 287, 41-47.

Ryan, R. P., Monchy, S., Cardinale, M., Taghavi, S., Crossman, L., Avison, M. B., Berg, G., van der Lelie, D. \& Dow, J. M. (2009). The versatility and adaptation of bacteria from the genus Stenotrophomonas. Nat Rev Microbiol 7, 514-525.

Schubert, S., Dalhoff, A., Stass, H. \& Ullmann, U. (2005). Pharmacodynamics of moxifloxacin and levofloxacin simulating human serum and lung concentrations. Infection 33, 15-21.

Whitby, P. W., Carter, K. B., Burns, J. L., Royall, J. A., LiPuma, J. J. \& Stull, T. L. (2000). Identification and detection of Stenotrophomonas maltophilia by rRNA-directed PCR. J Clin Microbiol 38, 4305-4309.

Wojnicz, D. \& Jankowski, S. (2007). Effects of subinhibitory concentrations of amikacin and ciprofloxacin on the hydrophobicity and adherence to epithelial cells of uropathogenic Escherichia coli strains. Int J Antimicrob Agents 29, 700-704. 